\title{
Enhancement of low-mass dileptons in ultraperipheral collisions
}

\author{
I. M. Dremin ${ }^{1, a}$, S. R. Gevorkyan ${ }^{2, b}$, D. T. Madigozhin ${ }^{2, c}$ \\ ${ }^{1}$ Lebedev Physical Institute, Moscow, Russia \\ 2 Joint Institute for Nuclear Research, Dubna, Russia
}

Received: 2 September 2020 / Accepted: 15 March 2021 / Published online: 2 April 2021

(C) The Author(s) 2021

\begin{abstract}
It is shown that production of low-mass $e^{+} e^{-}$pairs in ultraperipheral nuclear collisions is enhanced due to the Sommerfeld-Gamow-Sakharov (SGS) factor. This effect is especially strong near the threshold of creation of unbound $e^{+} e^{-}$-pairs with low masses in the two-photon fusion. Coulomb attraction of the non-relativistic components of such pairs may lead to the increased intensity of 511 $\mathrm{keV}$ photons. It can be recorded at the NICA collider and has some astrophysical implications. The analogous effect can be observed at LHC in dilepton production.
\end{abstract}

Production of $e^{+} e^{-}$-pairs in electromagnetic fields of colliding heavy ions was first considered by Landau and Lifshitz in 1934 [1]. It was shown that the total cross section of this process rapidly increases with increasing energy $E$ as $\ln ^{3} E$ in asymptotics. This is still the strongest energy dependence in particle physics. Moreover, the numerical factor $Z^{4} \alpha^{4}$ compensates in the total cross section the effect of the small electromagnetic coupling $\alpha$ for heavy ions with large charge $Z e$. Therefore, the ultraperipheral production of $e^{+} e^{-}$-pairs (as well as $\mu^{+} \mu^{-}$etc.) in ion collisions can become the dominant mechanism at very high energies. It is already widely studied at colliders. The heuristic knowledge of these processes is helpful in understanding some astrophysical phenomena as well.

Abundant creation of pairs with rather low masses is the typical feature of ultraperipheral interactions [2]. Dileptons are produced in grazing collisions of interacting ions where two photons from their electromagnetic clouds interact and a lepton pair is created. Two-photon fusion production of lepton pairs has been calculated with both the equivalent photon approximation proposed in [3,4] and via full lowest-order QED calculations [5-8] reviewed recently in [9]. According

\footnotetext{
a e-mail: dremin@lpi.ru

b e-mail: gevs@jinr.ru (corresponding author)

c e-mail: madigo@mail.ru
}

to the equivalent photon approximation, the spectra of dileptons created in ultraperipheral collisions can be obtained from the general expression for the total cross section

$\sigma_{u p}(X)=\int d x_{1} d x_{2} \frac{d n}{d x_{1}} \frac{d n}{d x_{2}} \sigma_{\gamma \gamma}(X)$.

Feynman diagrams of ultraperipheral processes contain the subgraphs of two-photon interactions leading to production of some final states $X$ (e.g., $e^{+} e^{-}$pairs). These blobs can be represented by the cross sections of these processes. Therefore, $\sigma_{\gamma \gamma}(X)$ in (1) denotes the total cross section of production of the state $X$ by two photons from the electromagnetic clouds surrounding colliding ions and $d n / d x_{i}$ describe the densities of photons carrying the share $x_{i}$ of the ion energy.

The distribution of equivalent photons with a fraction of the nucleon energy $x$ generated by a moving nucleus with the charge $Z e$ can be denoted as

$\frac{d n}{d x}=\frac{2 Z^{2} \alpha}{\pi x} \ln \frac{u(Z)}{x}$

if integrated over the transverse momentum up to some value (see, e.g., [10]). The physical meaning of the ultraperipherality parameter $u(Z)$ is the ratio of the maximum adoptable transverse momentum to the nucleon mass as the only massless parameter of the problem. Its value is determined by the form factors of colliding ions (see, e.g., [11]). It is clearly seen from Eq. (2) that soft photons with small fractions $x$ of the nucleon energy dominate in these fluxes.

The cross section $\sigma_{\gamma \gamma}(X)$ usually inserted in (1) in case of creation of the unbound dielectrons $X=e^{+} e^{-}$is calculated in the lowest order perturbative approach and looks $[10,12]$ as

$\sigma_{\gamma \gamma}(X)=\frac{2 \pi \alpha^{2}}{M^{2}}\left[\left(3-v^{4}\right) \ln \frac{1+v}{1-v}-2 v\left(2-v^{2}\right)\right]$,

where $v=\sqrt{1-\frac{4 m^{2}}{M^{2}}}$ is the velocity of the pair components in the pair rest system, $m$ and $M$ are the electron and dielectron masses, correspondingly. The cross section tends to 0 at 
the threshold of pair production $M=2 m$ and decreases as $\frac{1}{M^{2}} \ln M$ at very large $M$.

The distribution of masses $M$ of dielectrons is obtained after inserting Eqs (2), (3) into (1) and leaving free one integration there. One gets [2]

$$
\begin{aligned}
& \frac{d \sigma}{d M}=\frac{128(Z \alpha)^{4}}{3 \pi M^{3}}\left[\left(1+\frac{4 m^{2}}{M^{2}}-\frac{8 m^{4}}{M^{4}}\right)\right. \\
& \left.\times \ln \frac{1+\sqrt{1-\frac{4 m^{2}}{M^{2}}}}{1-\sqrt{1-\frac{4 m^{2}}{M^{2}}}}-\left(1+\frac{4 m^{2}}{M^{2}}\right) \sqrt{1-\frac{4 m^{2}}{M^{2}}}\right] \ln ^{3} \frac{u \sqrt{s_{n n}}}{M},
\end{aligned}
$$

where $\sqrt{s_{n n}}$ is the c.m.s. energy per a nucleon pair.

The perturbative expression for the cross section $\sigma_{\gamma \gamma}(X)$ of (3) can be generalized to include the non-perturbative effects crucial near the pair production threshold $M=2 m$. It happens to be possible for Coulomb interaction governing the behavior of the components of a pair. At the production point, the components of pairs with low masses close to $2 m$ move very slowly relative to one another. They are strongly influenced by the attractive Coulomb forces. In the non-relativistic limit, these states are transformed by mutual interactions of the components to effectively form a composite state whose wave function is a solution of the relevant Schroedinger equation. The normalization of Coulomb wave functions plays an especially important role at low velocities. It differs from the normalization of free motion wave functions used in the perturbative derivation of Eq. (3).

The amplitude $R_{C}$ of the process $\gamma \gamma \rightarrow e^{+} e^{-}$with account of the interaction between leptons is connected to the amplitude $R_{0}$ without the final state interaction by the relation

$R_{C}=\int \Psi_{f}(r) R_{0}(r) d^{3} r$

where $\Psi_{f}(r)$ is the wave function for bound (parapositronium) or unbound lepton pairs in the coordinate representation.

For lepton pairs in $S$-state (the orbital momentum $l=0$ ) the characteristic distances of the pair production are $1 / \mathrm{m}$, whereas the Coulomb interaction between leptons acts over the much larger distances $(1 / m \alpha$ in the bound state production and $1 / k$ for the unbound states $(k$ is the relative momentum). Therefore, the wave function can be considered as constant in (5) and one gets

$R_{C}=\Psi_{k S}(r=0) \int R_{0}(r) d^{3} r=\Psi_{k S}(r=0) R_{0}(p=0)$.

This relation is valid not only for bound states, but also for the creation of the unbound lepton pairs if $k r_{s} \ll 1$. Such factorization of matrix elements has been widely used in the dimesoatoms production [13]. It is useful for any process where the characteristic distances of pair production and of final state interactions are substantially different. The normalization of the unbound pair wave function reads [14]

$\left|\psi_{k S}(\mathbf{r}=0)\right|^{2}=\frac{\pi \xi}{\operatorname{sh}(\pi \xi)} e^{\pi \xi}=\frac{2 \pi \xi}{1-e^{-2 \pi \xi}} ; \quad \xi=\frac{2 \pi \alpha m}{k}$.

This is the widely used Sommerfeld-Gamow-Sakharov (SGS) factor [15-18] which unites the non-perturbative and perturbative matrix elements. It results in the so-called " $\frac{1}{v}$ law" of the enlarged outcome of the reactions with extremely low-mass pairs produced. This factor is described in the standard textbooks on non-relativistic quantum mechanics (see, e.g., [14]) and used in various publications (e.g., [19-22]). The Sakharov recipe of its account for production of $e^{+} e^{-}$pairs desctibed in [18] consists in direct multiplication of the differential distribution of Eq. (4) by the SGS-factor written as

$$
T=\frac{2 \pi \alpha}{v(1-\exp (-2 \pi \alpha / v))} .
$$

It enhances the contribution of the low-mass (low- $v$ ) pairs. Thus the proper distribution of dielectron masses in ultraperipheral processes is

$$
\begin{aligned}
\frac{d \sigma}{d M^{2}}= & \frac{128(Z \alpha)^{4}}{3 M^{4}} \frac{\alpha}{\sqrt{1-\frac{4 m^{2}}{M^{2}}}\left(1-\exp \left(-2 \pi \alpha / \sqrt{1-\frac{4 m^{2}}{M^{2}}}\right)\right)} \\
\times & {\left[\left(1+\frac{4 m^{2}}{M^{2}}-\frac{8 m^{4}}{M^{4}}\right) \ln \frac{1+\sqrt{1-\frac{4 m^{2}}{M^{2}}}}{1-\sqrt{1-\frac{4 m^{2}}{M^{2}}}}\right.} \\
& \left.-\left(1+\frac{4 m^{2}}{M^{2}}\right) \sqrt{1-\frac{4 m^{2}}{M^{2}}}\right] \ln ^{3} \frac{u \sqrt{s_{n n}}}{M}
\end{aligned}
$$

The distribution of the relative (in $e^{+} e^{-}$rest system) velocity $v$ is like

$$
\begin{gathered}
\frac{d \sigma}{d v^{2}}=\frac{16(Z \alpha)^{4}}{3 m^{2}}\left[\left(3-v^{4}\right) \ln \frac{1+v}{1-v}-2 v\left(2-v^{2}\right)\right] \\
\frac{\alpha}{v\left(1-\exp \left(\frac{-2 \pi \alpha}{v}\right)\right)} \ln ^{3} \frac{u \sqrt{s_{n n}\left(1-v^{2}\right)}}{2 m} .
\end{gathered}
$$

Let us remind that the velocity $v$ is related to the velocity of the positron $v_{+}$in the electron rest system as

$$
v^{2}=\frac{1-\sqrt{1-v_{+}^{2}}}{1+\sqrt{1-v_{+}^{2}}}
$$

so that $v_{+}=2 v$ at $v \rightarrow 0$ and both $v$ and $v_{+}$tend to 1 in the ultrarelativistic limit. The relative velocities $v$ and $v_{+}$are the relativistic invariants represented by the Lorentz-invariant masses $m$ and $M$. 


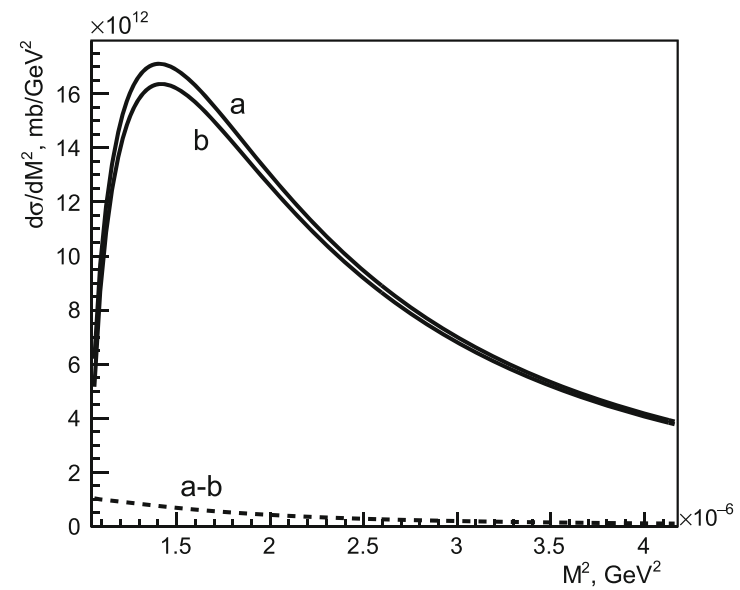

Fig. 1 The distribution of masses of dielectrons produced in ultraperipheral collisions at NICA energy $\sqrt{s_{n n}}=11 \mathrm{GeV}$ with (a) and without (b) account of the SGS-factor. Their difference $(\mathbf{a}, \mathbf{b})$ is shown by the dashed line. The region of small masses is shown in the right-hand side

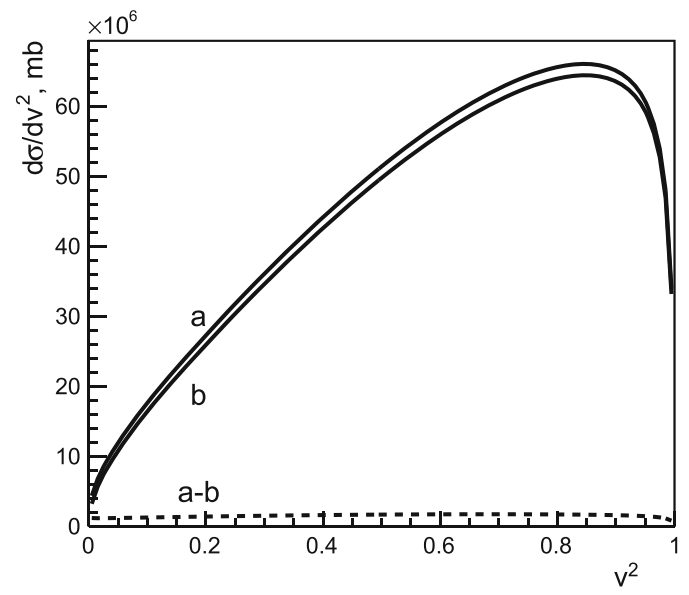

Fig. 2 The distribution of the relative velocities in dielectrons produced in ultraperipheral collisions at NICA energy $\sqrt{s_{n n}}=11 \mathrm{GeV}$ with (a) and without (b) account of the SGS-factor. Their difference $(\mathbf{a}, \mathbf{b})$ is

It has been shown in Ref. [2] (see Fig. 1 there) that the cross section of creation of unbound $e^{+} e^{-}$-pairs tends to zero at the threshold $M=2 m$ if the perturbative expression Eq. (3) is used. Account of the non-perturbative SGS-factor (8) in Eqs. (9) and (10) drastically changes the situation, especially at low masses $M$ and velocities $v$. In Figs. 1 and 2, we compare the yields of pairs with (curves a) and without (curves b) account of the SGS-factor at NICA energy 11 $\mathrm{GeV}$ as functions of masses $M$ and velocities $v$. It is clearly seen that the overall contribution due to the correction is not high. It amounts to about 4.6 percents at the peak of the $M^{2}$ distribution and 2.5 percents at the peak of the $v^{2}$ distribution. The integral contributions differ by 3.4 percents only.

The cross sections of ultraperipheral production of $e^{+} e^{-}$pairs are especially strongly enhanced at low masses $M$ (at

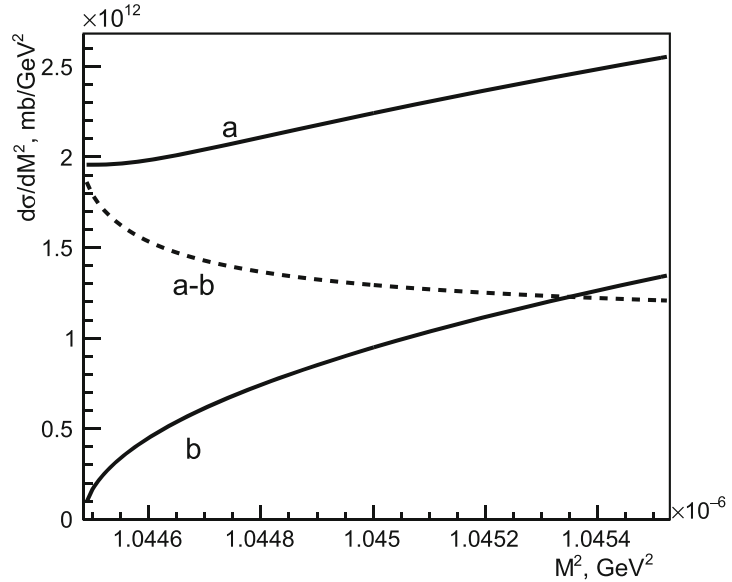

at the enlarged scale. Note the factor $10^{-6}$ at the abscissa scale which reduces it to $\mathrm{MeVs}$. The $\mathrm{GeV}$-scale is chosen here for further direct comparison with scales of the muon-pairs production at LHC shown in Fig. 3

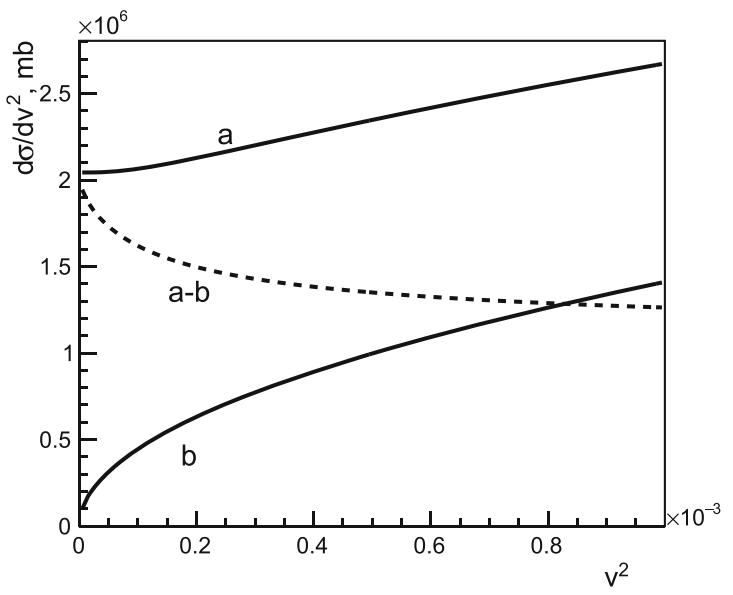

shown by the dashed line. The velocities for the region of small masses are shown in the right-hand side. Note the factor $10^{-3}$ at the abscissa scale

low relative velocities $v$ ) compared to their perturbative values (marked by b). It is clearly seen in the righthand sides of Figs. 2 and 2 which demonstrate the region near the threshold $M=2 m$. Surely, the cross section would tend to zero at the threshold $M=2 m$ due to the energy-momentum conservation laws not fully respected by the simplified SGS-recipe. However, it must happen in the tiny region near the threshold and can be neglected in integral estimates.

The situation with production of muon pairs is similar. We demonstrate it in Fig. 3 by plotting the distribution of masses of muon pairs produced in ultraperipheral collisions at LHC energy $\sqrt{s_{n n}}=5.02 \mathrm{TeV}$. There is no principal difference between the general shapes in Fig. 3 and Figs. 1 and 2 . The scales on both axes are just changed. The main result 


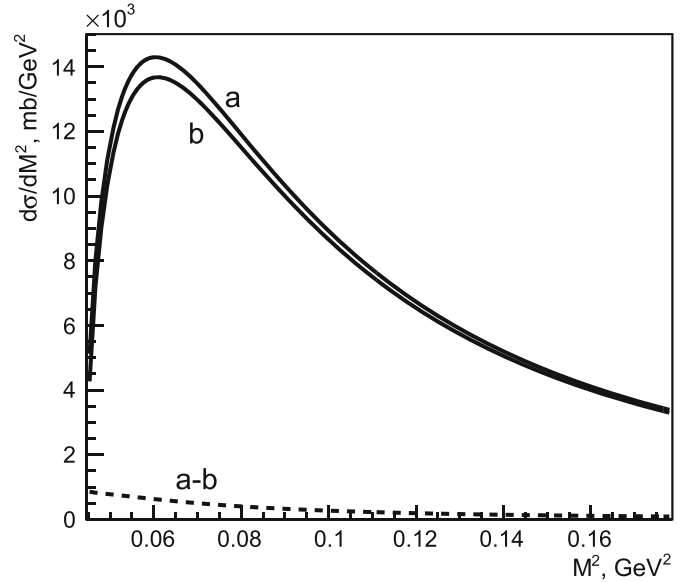

Fig. 3 The distribution of masses of muon pairs produced in ultraperipheral collisions at LHC energy $\sqrt{s_{n n}}=5.02 \mathrm{TeV}$ with (a) and without (b) account of the SGS-factor. Their difference $(\mathbf{a}, \mathbf{b})$ is shown by the

about the enhanced low-mass pairs persists at higher energies as well.

The $e^{+} e^{-}$-pairs with low masses would be of no special interest if their final products are electrons and positrons. The background is such high that they are buried among many charged products of particle collisions. However the mutual attraction of these components can result in their annihilation. Two $\gamma$-quanta are created with definite energy $M / 2$ in the rest system of a pair. This is the clear signature for their detection. The energy distribution of quanta is peaked at $511 \mathrm{keV}$ in NICA experiments as shown in Ref. [2] for the parapositronia decays. The observation of such quanta can signal the onset of their ultraperipheral production there.

However, it is not excluded that the pairs with masses somewhat exceeding the threshold $M=2 m$ can also annihilate to two quanta with approximately the same energy. The peaks will be slightly widened. The fate of the low-mass pairs with slow relative motion of its components is hard to predict. The strong Coulomb attraction is crucial.

The SGS-effect arises when an attractive interaction between the non-relativistic particles significantly distorts their wave function, such that they have a larger probability to undergo annihilation. It has been claimed in Ref. [20] that the quantum field theory Bethe-Salpeter equation in the ladder approximation provides the leading non-perturbative solution, with perturbative corrections coming from higher order diagrams. The non-relativistic nature of the pair of annihilating particles separates the short-distance annihilation process (taking place at distances up to $\mathrm{O}(1 / \mathrm{m})$ ) from the long-distance interactions characterized by the Bohr radius of the pair, responsible for the SGS-effect. The transition is determined by their ratio which plays a role of the small parameter.

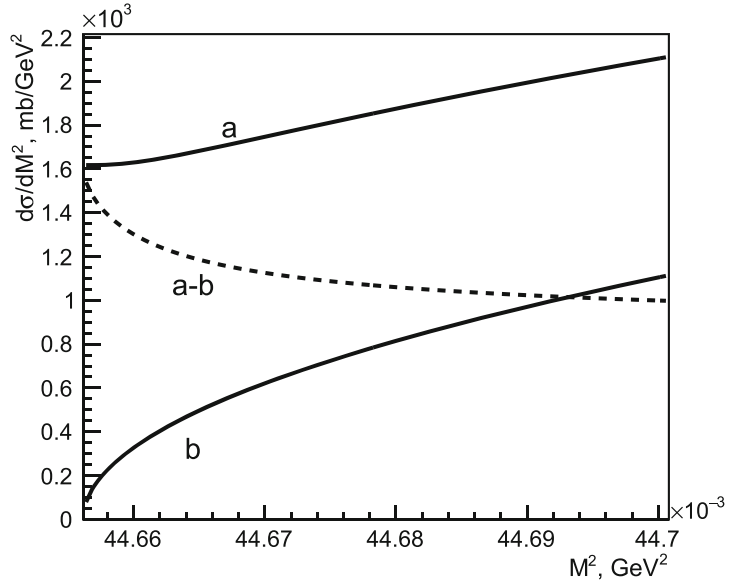

dashed line. The region of small masses is shown in the right-hand side at the enlarged scale. Note the factor $10^{-3}$ at the abscissa scale

An anomalous low-mass dilepton excess demonstrated above in Figs. 1, 2 and 3 attracts much attention as a mechanism to boost the annihilation rates. The ultraperipheral production of parapositronia is about $10^{6}$ times lower than the supply of unbound pairs. The integral contribution of the lowmass unbound pairs exceeds the parapositronia decay effect by several orders of magnitude.

The enhancement of low-mass $e^{+} e^{-}$-pairs can be extremely important for understanding some observations of abundant production of the $511 \mathrm{keV} \gamma$-quanta during the thunderstorms in the Earth atmosphere [23] and a distinctive peak at this energy in signals from the Universe [24]. The parapositronia at rest may produce such peaks. It was speculated in Ref. [2] that the common origin of these effects can be prescribed to the formation of the dense electron-positron clouds by strong electromagnetic fields. The kinetics of electrons and positrons in the clouds should admit (probably, via intense thermal infrared radiation) their cooling down to form parapositronia or low-mass unbound pairs which would produce pairs of photons with energies close to 511 $\mathrm{keV}$. The relative motion of the components of the low-mass pairs produced in ultraperipheral processes is non-relativistic $\left(v \sim 10^{-1}-10^{-2}\right.$ ) as demonstrated in the right-hand side of Fig. 2. Thus it takes a long time for them to separate and, when traveling from distances about the classical electron radius of the order of the inverse electron mass to the Bohr radius which is more than two orders of magnitude larger, they can annihilate into two photons due to attraction via exchange by the long-wave infrared radiation.

One should take into account that before their transverse separation the pairs travel a long way in the galactic surroundings after being formed by high energy jets of matter during the star explosions. That increases the annihilation 
probability. These problems will be considered in a separate publication.

As shown in [2] the peaks can also be widened due to the motion of produced pairs. Creation of low-mass pairs would give rise to the shift of the peak with its further widening. The widths of the peaks coming from different regions of the Milky Way vary from $2.5 \mathrm{keV}$ to $3.5 \mathrm{keV}$ according to the results of Ref. [24]. These widths may correspond to the admissible intervals of masses $M$ ranging from $2 m=1.022$ $\mathrm{MeV}$ to $1.027 \mathrm{MeV}$ and $1.029 \mathrm{MeV}$ correspondingly and can be ascribed to annihilation of $e^{+} e^{-}$-pairs with such masses. Integrating the plot a in Fig. 1 within these intervals of masses one gets the cross sections equal to $40165 \mathrm{mb}$ and 63296 mb which are much larger than the cross section of the parapositronium production about $20 \mathrm{mb}$. Therefore, the outcome of $\gamma$-quanta with energies near $511 \mathrm{keV}$ may become more abundant than just from decays of parapositronia. The behavior of pairs with masses close to $2 \mathrm{~m}$ asks for the special consideration.

Studies at the NICA collider can help in getting the quantitative results about properties of $e^{+} e^{-}$-pairs created in strong electromagnetic fields of heavy ions during their ultraperipheral collisions.

Acknowledgements The work of I.D. was supported by the RFBR project 18-02-40131.

Data Availability Statement This manuscript has no associated data or the data will not be deposited. [Authors' comment: There are no associated data available.]

Open Access This article is licensed under a Creative Commons Attribution 4.0 International License, which permits use, sharing, adaptation, distribution and reproduction in any medium or format, as long as you give appropriate credit to the original author(s) and the source, provide a link to the Creative Commons licence, and indicate if changes were made. The images or other third party material in this article are included in the article's Creative Commons licence, unless indicated otherwise in a credit line to the material. If material is not included in the article's Creative Commons licence and your intended use is not permitted by statutory regulation or exceeds the permitted use, you will need to obtain permission directly from the copyright holder. To view a copy of this licence, visit http://creativecomm ons.org/licenses/by/4.0/.

Funded by SCOAP ${ }^{3}$.

\section{References}

1. L.D. Landau, E.M. Lifshitz, Phys. Zeit. Sowjet. 6, 244 (1934)

2. I.M. Dremin, Universe 6(7), 94 (2020); arXiv:2006.12033

3. C.F.V. Weizsäcker, Z. Phys. 88, 612 (1934)

4. E.J. Williams, Phys. Rev. 45, 725 (1934)

5. G. Racah, Nuovo Cim. 14, 93 (1937)

6. V.M. Budnev, I.F. Ginzburg, G.V. Meledin, V.G. Serbo, Phys. Rep. 15, 181 (1975)

7. E. Bartosh, S.R. Gevorkyan, E.A. Kuraev, N.N. Nikolaev, Phys. Rev. A 66, 042720 (2020)

8. S.R. Gevorkyan, E.A. Kuraev, J. Phys. G 29, 1 (2013)

9. I.M. Dremin, Phys. Usp. 190, 811 (2020)

10. V.B. Berestetsky, E.M. Lifshitz, L.P. Pitaevsky, Kvantovaya Electrodinamika (Fizmatlit, Moscow, 2001)

11. M.I. Vysotsky, E.V. Zhemchugov, Phys. Usp. 189, 975 (2019)

12. G. Breit, J.A. Wheeler, Phys. Rev. 46, 1087 (1934)

13. L. Afanasyev, S. Gevorkyan, O. Voskresenskaya, Eur. Phys. J. A $\mathbf{5 3}, 78$ (2017)

14. L.D. Landau, E.M. Lifshitz, Kvantovaya Mechanika, Nerelyativistskaya Teoriya, 2 izdanie, (134.11) (Fizmatlit, Moscow ,1963) (translated in: Quantum Mechanics (Pergamon Press, Oxford, 1977))

15. A. Sommerfeld, Atombau und Spectralinien (F. Vieweg und Sohn, Brunswick, 1921)

16. G. Gamow, Z. Phys. 51, 204 (1928)

17. A. Sommerfeld, Ann. Phys. (Leipz.) 403, 257 (1931)

18. A.D. Sakharov, Zh. Eksp. Teor. Fiz. 18, 631 (1948) (Reprinted in Sov. Phys. Usp. 34, 375 (1991))

19. V.N. Baier, V.S. Fadin, Sov. Phys. JETP 30, 127 (1970)

20. R. Iengo, JHEP 05, 024 (2009). arXiv:0902.0688

21. S. Cassel, J. Phys. G 37, 105009 (2010). arXiv:0903.5307

22. A.B. Arbuzov, T.V. Kopylova, JHEP 04, 009 (2012). arXiv: 1111.4308

23. J.R. Dwyer et al., J. Plasma Phys. 81, 475810405 (2015). arXiv: 1505.03782

24. T. Siegert et al., A\&A 586, A84 (2016). arXiv:1512.00325 